\title{
Low Accelerating Voltage Scanning Transmitted Electron Microscope: Imaging, Diffraction, X-ray Microanalysis, and Electron Energy-Loss Spectroscopy at the Nanoscale
}

\author{
Hendrix Demers ${ }^{1}$, Nicolas Brodusch ${ }^{1}$, and Raynald Gauvin ${ }^{1}$ \\ 1. Department of Mining and Materials Engineering, McGill University, Montreal, Quebec, Canada.
}

For developing new technologies, it is important to characterize materials at the nanoscale. To achieve high resolution, a field emission scanning electron microscope (FE-SEM) with low voltage scanning transmitted electron microscope mode (STEM) was developed. The HITACHI SU-9000 provides low accelerating voltage, deceleration mode, and energy-filtering of the electron signals (secondary and backscattered electrons), low voltage STEM bight-field (BF) and dark-field (DF) imaging capability are possible on these instruments to allow the characterization of materials at the nanoscale with various types of contrasts. Furthermore, the instrument has diffraction analysis, electron energy loss spectrometry (EELS), and energy dispersive spectrometry (EDS) systems, which allow crystallographic and chemical characterization at the nanoscale materials.

Figure 1 shows an example of high resolution imaging of an AA 2099 Al-Li alloy in BF and DF imaging modes at low voltage $(30 \mathrm{kV})$. One of the advantages of the low accelerating voltage used is the increase of the contrast observed, which is very important when the characterized phases containing lithium and the difference in mass density is very small. Another problem with $\mathrm{Li}$ alloy is the electron probe damage, which is minimized when using low accelerating voltage by reducing the electron dose.

Figure 2 shows examples of diffraction analysis and imaging. Convergent-beam electron diffraction (CBED) of an AA 2099 Al-Li alloy was done and the dynamic effect were observed inside the CBED pattern disks. Recently the low voltage CBED was used to determine the number of graphene layers [1]. Lattice imaging of a multi wall carbon nanotube in BF mode was also obtained at low voltage $(30 \mathrm{kV})$. Previous study has explained the method and give other sample examples [3]. The Fourier transform of the lattice fringes indicates a distance of $0.34 \mathrm{~nm}$ which is in agreement with previous observation [4].

The high resolution imaging is complemented by two analytical systems: electron energy loss spectrometry (EELS) and energy dispersive spectrometry (EDS). Examples of low voltage EELS were previously presented to validate the possibility of low voltage EELS [1,2]. Figure 3 shows an example of high resolution $\mathrm{x}$-ray EDS microanalysis. A line scan across a $\mathrm{T}_{1}$ precipitate in an AA 2099 Al-Li alloy was recorded. The spatial resolution on the DF micrograph is between 2.0 and $2.5 \mathrm{~nm}$ and around $3.0 \mathrm{~nm}$ on the x-ray line scan. The low concentration of $\mathrm{Zn}$ in the $\mathrm{T}_{1}\left(\mathrm{Al}_{2} \mathrm{CuLi}\right)$ was clearly observed. Furthermore, the decrease of $\mathrm{Al}$ concentration in the $\mathrm{T}_{1}$ phase versus the $\mathrm{Al}$ matrix was also observed. We are currently working to acquire EELS and EDS data simultaneously to clearly observe the complete composition of each Al-Li alloy phase as the low concentration Zn cannot be detected with EELS [5] and Li is difficult to detect with EDS.

These examples show the high resolution imaging and analysis possibility of low voltage STEM of instrument like SU-9000 for characterization of nanoscale materials and also minimizing the electron probe damage by reducing the dose used. 


\section{References:}

[1] T. Sunaoshi et al, Microscopy and Microanalysis 22 (2016), pp. 604-605.

[2] Y. Yamazawa et al, Microscopy and Microanalysis 22 (2016), pp. 50-51.

[3] M. Konno et al, Ultramicroscopy 145 (2014) pp. 28-35.

[4] V. Van Ngo et al, Microscopy Today 15 (2007) pp. 12-16.

[5] M.-F. Guinel et al, Journal of Microscopy 255 (2014) pp. 128-137.

[6] This work was supported by Hitachi High-Technologies Canada Inc.

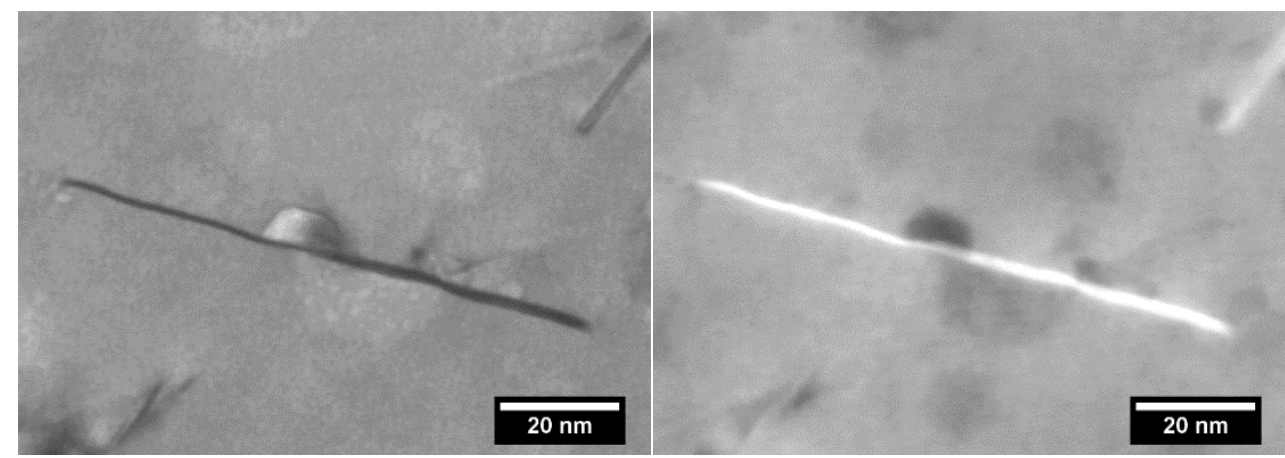

Figure 1. Example of high resolution imaging with an AA 2099 Al-Li alloy. (left) Bright-field micrograph; (right) Dark-field micrograph.
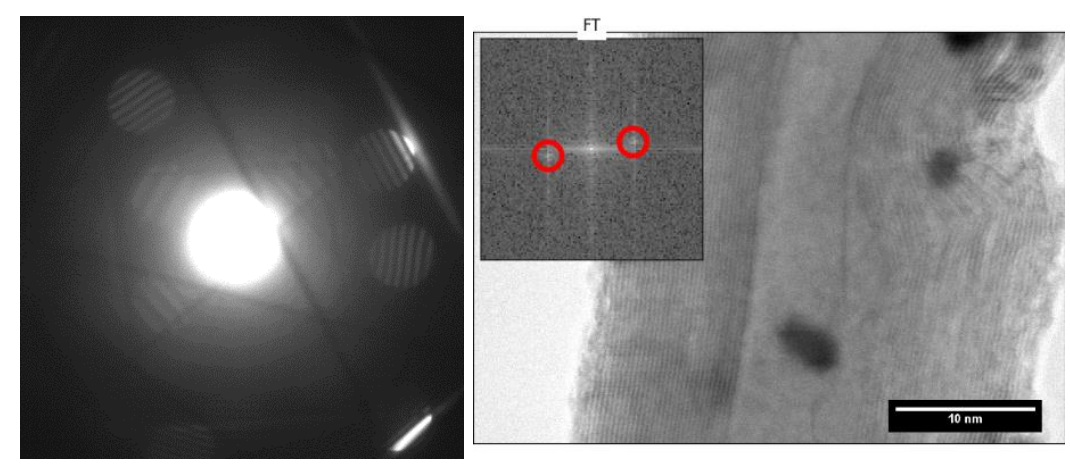

Figure 2. Example of diffraction analysis and imaging. (left) Convergent-beam electron diffraction of an AA 2099 Al-Li alloy; (right) Lattice imaging of a multi-wall carbon nanotube, the inset shows a Fourier transform of the interference fringes and the 2 red circles give a spatial resolution of $0.34 \mathrm{~nm}$.
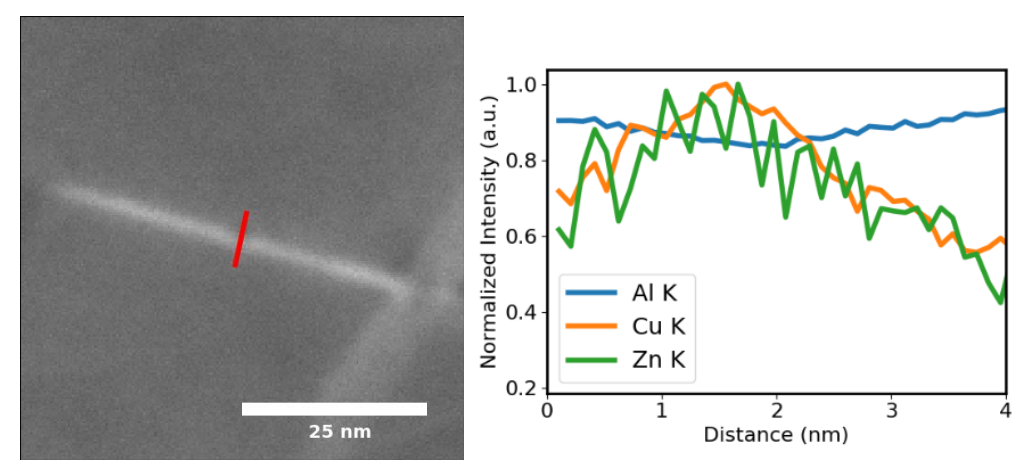

Figure 3. Example of high resolution $x$-ray microanalysis. (left) dark-field micrograph showing the location of the $\mathrm{x}$-ray line scan (right). The line scan is over a $\mathrm{T}_{1}$ microstructure of an AA $2099 \mathrm{Al}-\mathrm{Li}$ alloy. The spatial resolution on the DF micrograph is between 2.0 and $2.5 \mathrm{~nm}$ and around $3.0 \mathrm{~nm}$ on the $\mathrm{x}$-ray line scan. 